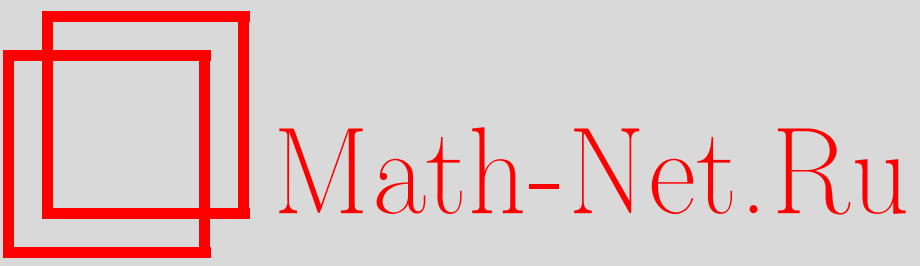

М. М. Глухов, К анализу некоторых систем открытого распределения ключей, основанных на неабелевых группах, Матем. вопр. криптогр., 2010, том 1, выпуск 4, 5-22

DOI: https://doi.org/10.4213/mvk18

Использование Общероссийского математического портала Math-Net.Ru подразумевает, что вы прочитали и согласны с пользовательским соглашением

http: //www . mathnet.ru/rus/agreement

Параметры загрузки:

IP : 18.209 .158 .208

26 апреля 2023 г., 17:30:42 
УДК 512.54.05

\title{
К анализу некоторых систем открытого распределения ключей, основанных на неабелевых группах
}

\author{
Глухов М. М.
}

Академия криптографии Российской Федерации, Москва

Получено 20.Х.2010

В работе анализируются некоторые криптосистемы открытого распределения ключей, основанные на композиции проблем сопряженности и логарифмирования в неабелевых (некоммутативных) группах, определяемых на множестве $\left(\boldsymbol{Z}_{p}\right)^{4}$. Для рассмотренных схем доказано, что верхняя оценка сложности задачи нахождения секретного ключа по порядку не превосходит сложности проблемы дискретного логарифмирования в циклической подгруппе мультипликативной группы поля $\boldsymbol{Z}_{p}$ или его квадратичного расширения.

Ключевые слова: криптосистема, открытый ключ, неабелева группа, проблема сопряженности, проблема дискретного логарифмирования, жорданова матрица

An analysis of some key distribution public systems based on non-abelian groups

\section{M. Gluhov}

Academy of Cryptography of Russian Federation, Moscow

\begin{abstract}
We consider some cryptosystems for public distribution of keys based on the composition of the conjugacy and discrete logarithm problems for non-abelian (non-commutative) groups constructed on $\left(\boldsymbol{Z}_{p}\right)^{4}$. It is proved that for these schemes the upper bound of complexity of breaking the secret key does not exceed (in the order) the complexity of discrete logarithm problem for cyclic subgroup of the multiplicative group of the field $\boldsymbol{Z}_{p}$ or its quadratic extension.
\end{abstract}

Key words: cryptosystem, public key, non-abelian group, conjugacy problem, discrete logarithm ptoblem, Jordan matrix

Citation: Mathematical Aspects of Cryptography, 2010, vol. 1, no. 4, pp. 5-22 (Russian). 


\section{1. Введение}

Первые криптосистемы открытого распределения ключей типа ДиффиХеллмана основывались на проблеме дискретного логарифмирования в циклических подгруппах абелевых групп. При этом в качестве групп выбирались мультипликативные группы конечных полей и колец вычетов, группы эллиптических кривых над конечными полями, группы классов идеалов колец целых алгебраических чисел и др.

В 1997 г. появилась работа [13], в которой было доказано, что проблема дискретного логарифмирования имеет полиномиальную сложность при ее решении на квантовом компьютере. Эта работа инициировала исследования по отысканию других сложных математических проблем, которые можно было бы использовать при построении асимметричных криптосистем. В 1998-2001 годах появились работы [6,7,16,17], в которых предлагались системы открытого распределения ключей, основанные на проблеме сопряженности в некоммутативных группах. В двух первых из них использовались матричные группы, в последних двух - группы кос. В последующих работах было показано, что все такие системы являются уязвимыми. Подробную историческую справку об этих системах и соответствующую библиографию можно найти в работах $[14,15]$.

В 2001 г. были опубликованы работы [10,11], в которых строились асимметричные криптосистемы, основанные на проблеме дискретного логарифмирования в группах внутренних автоморфизмов полупрямых произведений групп $S L\left(2, \boldsymbol{Z}_{p}\right)$ и $\boldsymbol{Z}_{p}$ в [10] и $G L\left(2, \boldsymbol{Z}_{p}\right)$ и $\boldsymbol{Z}_{p}$ в [11]. Фактически в них уже использовалась определенная композиция двух проблем в некоммутативных группах: проблемы сопряженности и проблемы логарифмирования. Такие криптосистемы были названы авторами MOR-системами. В работе [14] анализируется более общая MOR-система, в которой используется полупрямое произведение групп $G L(2, R)$ и $\boldsymbol{Z}_{n}$, где $R$ - коммутативное кольцо с единицей. Показано, что рассматриваемые MOR-системы уязвимы по специально подобранному шифртексту, если проблема Диффи-Хеллмана решается эффективно в циклической группе автоморфизмов, порожденной внутренним автоморфизмом группы $G$.

В 2007 г. в работе [12] была предложена схема открытого распределения ключей, основанная также на композиции двух указанных проблем теории групп. В [12] используются некоммутативная группа $G$ и ее коммутативная подгруппа $G_{1}$. Открытым ключом являются элемент $\theta \in G$ и матричное представление $\varphi$ группы $G$ над конечным полем $F$, а протокол получения общего секретного ключа выглядит следующим образом.

Абонент А выбирает случайно секретные элементы $\alpha \in G_{1}, r \in N$, вычисляет $\alpha \theta^{r} \alpha^{-1}$, переходит к матрице $A Q^{r} A^{-1}$, где $A=\varphi(\alpha), Q=\varphi(\theta)$, и высылает ее абоненту В. 
Абонент В аналогично выбирает элементы $\beta \in G_{1}, s \in N$, находит матрицу $B Q^{s} B^{-1}$ и высылает ее абоненту A.

Теперь А и В вычисляют соответственно матрицы $K_{A}=A\left(B Q^{s} B^{-1}\right)^{r} A^{-1}$ и $K_{B}=B\left(A Q^{r} A^{-1}\right)^{s} B^{-1}$.

Так как $A B=B A$, то $K_{A}=K_{B}=K$. Матрица $K$ и служит основой для получения общего секретного ключа абонентов А, В.

Авторы отмечают, что при построении указанной криптосистемы они использовали идеи работы [2] (см. также [3]).

В работах $[4,5,8,9]$ предлагается ряд схем открытого распределения ключей, аналогичных схеме работы [12]. Их специфика заключается в выборе используемых некоммутативных групп, определяемых на векторах линейных пространств небольших размерностей $n$ над конечным полем. При этом основное внимание уделяется случаю $n=4$ (исходя из возможностей более простой реализации схем и более простого способа построения нужных неабелевых групп).

Авторы предполагают, что задача вскрытия ключей в таких системах не сводится к проблеме дискретного логарифмирования в циклической группе конечного поля. В связи с этим делается также предположение о том, что «нахождение логарифма в некоммутативной группе имеет экспоненциальную сложность» (см. [4, стр. 244]).

Цель данной работы заключается в анализе криптосистем, предлагаемых в работах $[4,5,8,9]$ при использовании групп, определяемых на вектоpax пространства размерности $n=4$ над полем $\boldsymbol{Z}_{p}$. Доказано, что верхняя оценка их криптографической стойкости по порядку не превосходит сложности проблемы дискретного логарифмирования в циклической подгруппе простого порядка $q$ мультипликативной группы поля $\boldsymbol{Z}_{p}$ или его квадратичного расширения. При этом $q$ является делителем числа $p-1$ или $p+1$.

Подробное описание анализа будет проведено на примере одной схемы из работы [5]. Для всех остальных схем из работ $[4,5,8,9]$ при $n=4$ рассуждения аналогичны, и основной вывод тот же.

При изложении результатов будут использоваться следующие обозначения:

$\boldsymbol{Z}_{p}$ - поле классов вычетов по простому модулю $p$ при отождествлении его элементов с числами $0,1, \ldots, p-1$, арифметические операции над которыми проводятся по модулю $p$ и обозначаются теми же знаками, что и операции над числами;

$\boldsymbol{Z}_{p}^{*}$ - мультипликативная группа поля $\boldsymbol{Z}_{p}$;

$|G|$ — порядок группы $G$;

$\operatorname{ord}(g)$ - порядок элемента $g$ в групе $G$ или $\boldsymbol{Z}_{p}^{*}$;

$J(A)$ - жорданова форма матрицы $A$.

Все необходимые сведения из алгебры можно найти, например, в учебнике [1]. 


\section{2. Описание криптосистемы и постановка задачи}

Предложенные в работах $[4,5,8,9]$ криптосистемы открытого распределения ключей основаны на композиции двух сложных проблем теории групп: проблемы сопряженности в некоммутативной группе и проблемы дискретного логарифмирования в ее циклической подгруппе. При этом под проблемой сопряженности в группе понимается проблема распознавания сопряженности элементов и нахождения сопрягающего (трансформирующего) элемента.

В предлагаемых системах для построения общего ключа абоненты А и В выбирают общую неабелеву группу $G$ и ее элемент $g$ достаточно большого простого порядка.

Далее протокол выработки общего ключа выглядит следующим образом.

1. Абонент А выбирает случайно элемент $X_{1} \in G$ с условием $X_{1} g \neq g X_{1}$ и число $x_{1} \in Z_{p}$, вычисляет элемент $Y_{1}=X_{1} g^{x_{1}} X_{1}^{-1}$ и отправляет его абоненту В.

2. Абонент В выбирает случайно элемент $X_{2} \in G$ с условием $X_{2} g \neq g X_{2} \quad$ и число $x_{2} \in Z_{p}$, вычисляет элемент $Y_{2}=X_{2} g^{x_{2}} X_{2}^{-1}$ и отправляет его абоненту А.

3. Абонент А вычисляет $K_{1}=\left(X_{1} Y_{2} X_{1}^{-1}\right)^{x_{1}}=X_{1} X_{2} g^{x_{2} x_{1}} X_{2}^{-1} X_{1}^{-1}$.

4. Абонент В вычисляет $K_{2}=\left(X_{2} Y_{1} X_{2}^{-1}\right)^{x_{2}}=X_{2} X_{1} g^{x_{1} x_{2}} X_{1}^{-1} X_{2}^{-1}$.

Если $X_{1}, X_{2}$ выбраны так, что $X_{1} X_{2}=X_{2} X_{1}$, то $K_{1}=K_{2}=K$, и $K$ есть общий ключ абонентов А, В.

При выполнении этого протокола естественно возникает техническая проблема выбора коммутирующих между собой и не коммутирующих c $g$ элементов $X_{1}, X_{2}$. Эта проблема преодолевается путем выбора $X_{1}, X_{2}$ степенями одного и того же элемента $h$. Тогда вместо случайного выбора $X_{1}, X_{2}$ абоненты A, В выбирают соответственно случайные натуральные числа $y_{1}, y_{2}$, меньшие порядка элемента $h$, и в качестве $X_{1}, X_{2}$ используют соответственно элементы $h^{y_{1}}, h^{y_{2}}$ группы $G$. При этом элемент $h$ и числа $y_{1}, y_{2}$ выбираются так, чтобы $h$ имел достаточно большой простой порядок, а элементы $h^{y_{1}}, h^{y_{2}}$ не коммутировали с $g$. Общим секретным ключом будет $h^{y_{1}+y_{2}} g^{x_{1} x_{2}} h^{-\left(y_{1}+y_{2}\right)}$.

Рассмотрим конкретный пример, в котором требуемая некоммутативная группа $G$ строится следующим образом (см. [5], Таблица 2 при $B=$ $=C=1)$. Выбирается большое простое число $p$ и рассматривается кольцо вычетов $\boldsymbol{Z}_{p}=\{0,1, \ldots, p-1\}$. На множестве $\left(\boldsymbol{Z}_{p}\right)^{4}$ определяются операции сло- 
жения и умножения. Сложение векторов $\alpha=(a, b, c, d)$ и $\chi=(x, y, z, w)$ производится покомпонентно, а умножение - по правилу

$$
\begin{gathered}
\alpha \chi=(a, b, c, d)(x, y, z, w)= \\
=(a x-\varepsilon b y-\varepsilon c z-d w, b x+a y-d z+c w, c x+d y+a z-b w, \\
d x-\varepsilon c y+\varepsilon b z+a w),
\end{gathered}
$$

где $\varepsilon \in \boldsymbol{Z}_{p}$ - структурный параметр. Заметим, что при $\varepsilon=1$ указанное правило умножения совпадает с правилом умножения кватернионов $a+b i+c j+d k$ с целыми коэффициентами с последующим приведением результатов операций над числами по модулю $p$. В общем случае (при любом $\varepsilon \in \boldsymbol{Z}_{p}$ ) введенная операция умножения соответствует следующей таблице умножения базисных единиц (векторов)

$$
\begin{aligned}
& e=(1,0,0,0), i=(0,1,1,1), j=(0,0,1,0), k=(0,0,0,1) . \\
& \begin{array}{ccccc}
\times & e & i & j & k \\
e & e & i & j & k \\
i & i & -\varepsilon e & \varepsilon k & -j \\
j & j & -\varepsilon k & -\varepsilon e & i \\
k & k & j & -i & -e
\end{array}
\end{aligned}
$$

Таблица 1

Указанная операция умножения при любом $\mathcal{E} \in \boldsymbol{Z}_{p}$ ассоциативна и дистрибутивна относительно сложения, но не коммутативна. В итоге получены некоммутативная полугруппа $H$ с единицей $e=(1,0,0,0)$ и линейная алгебра $\Omega$ размерности 4 над полем $\boldsymbol{Z}_{p}$ (при естественно определенной операции умножения векторов на элементы из $\boldsymbol{Z}_{p}$ ). В качестве искомой группы $G$ берется множество всех обратимых элементов полугруппы $H$. Авторами вычислен порядок группы $G$, а именно, доказаны следующие утверждения.

Утверждение 1.1. Если $\mathcal{E} \neq 0$ и $p \equiv 1(\bmod 4)$, mo $|G|=p(p-1)\left(p^{2}-1\right)$.

Утверждение 1.2. Если $\varepsilon=0$, то:

$|G|=p^{2}\left(p^{2}-1\right) n p u \quad p \equiv 3(\bmod 4) u|G|=p^{2}(p-1)^{2} n p u p \equiv 1(\bmod 4)$.

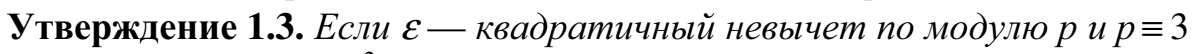
$(\bmod 4), m o|G|=p(p-1)\left(p^{2}-1\right)$.

\section{2. Дополнительные сведения о строении группы $G$}

Всюду далее под $H$ и $G$ будут пониматься построенные выше полугруппа и группа на множестве элементов вида $\alpha=(a, b, c, d)$, где $a, b, c, d \in \boldsymbol{Z}_{p}$. 
Утверждение 2.1. Полугруппа Н изоморфна подполугруппе матрии порядка 4 над полем $\boldsymbol{Z}_{p}$. Изоморфизмом является отображение $\varphi$, сопоставляюшее каждому элементу $\alpha=(a, b, c, d) \in H$ матрииу

$$
A_{\alpha}=\left(\begin{array}{cccc}
a & -\varepsilon b & -\varepsilon c & -d \\
b & a & -d & c \\
c & d & a & -b \\
d & -\varepsilon c & \varepsilon b & a
\end{array}\right) .
$$

Доказательство утверждения 2.1 проводится путем непосредственной проверки равенства $A_{\alpha} A_{\beta}=A_{\alpha \beta}$. Инъективность отображения $\varphi$ очевидна.

Определение 1. Элемент $a^{2}+\varepsilon b^{2}+\varepsilon c^{2}+d^{2} \in Z_{p}$ будем называть нормой элемента $\alpha=(a, b, c, d)$ и обозначать через $N(\alpha)$.

Утверждение 2.2. Элемент $\alpha=(a, b, c, d) \in H$ обратим тогда и только тогда, когда $N(\alpha) \neq 0$. При этом

$$
\alpha^{-1}=\frac{1}{N(\alpha)}(a,-b,-c,-d) .
$$

Для доказательства условия обратимости элемента $\alpha$ достаточно заметить, что определитель

$$
\operatorname{det}\left(A_{\alpha}\right)=\left(a^{2}+\varepsilon b^{2}+\varepsilon c^{2}+d^{2}\right)^{2}=(N(\alpha))^{2},
$$

и воспользоваться утверждением 2.1. При выполнении условия обратимости равенство (1) доказывается непосредственной проверкой.

Следующее утверждение отвечает на естественный вопрос о возможных значениях норм элементов из $G$.

Утверждение 2.3. Норма $N(\alpha)$ в зависимости от значения $\alpha \in G$ принимает любое значение из группы $\mathbf{Z}_{p}^{*}$ и удовлетворяет условию

$$
\forall \alpha, \beta \in G: N(\alpha \beta)=N(\alpha) N(\beta) \text {. }
$$

Доказательство. Условие (3) следует из (2). Найдем значения норм элементов $\alpha=(a, b, c, d) \in G$. Рассмотрим элемент $u=a^{2}+\varepsilon b^{2}+\varepsilon c^{2}+d^{2} \in Z_{p}$. Введем обозначение $M=\left\{a^{2}+d^{2}: a, d \in Z_{p}\right\}$ и докажем, что $Z_{p} \subseteq M$. Заметим, что $0 \in M$ и любой квадратичный вычет $r$ по модулю $p$ также содержится в $M$, поскольку $r=a^{2}$ при некотором $a \in Z_{p}$ и выполняется равенство $a^{2}=a^{2}+0^{2}$. Остается рассмотреть квадратичные невычеты по модулю $p$. Допустим, что $M$ содержит лишь 0 и квадратичные вычеты. Так как $a^{2}, b^{2}-$ квадратичные вычеты, то из указанного предположения следовало бы, что 
множество $M$ замкнуто относительно операции сложения. А так как оно конечно и замкнуто относительно умножения, то оно было бы подполем поля $Z_{p}$, что невозможно в силу простоты поля $Z_{p}$. Значит, $M$ содержит хотя бы один квадратичный невычет $s$. Пусть $s=a^{2}+d^{2}$ при некоторых фиксированных $a, d \in Z_{p}$. Умножив последнее равенство на любой квадратичный вычет $r=u^{2}$, получим равенство $s r=(a u)^{2}+(d u)^{2}$. Отсюда следует, что все квадратичные невычеты также содержатся в $M$. В итоге доказано, что $Z_{p} \subseteq M$ и, значит, $Z_{p}=M$. Тогда, если $\varepsilon=0$, величина $N(\alpha)=a^{2}+d^{2}$ в зависимости от $a, d$ будет принимать любые значения из $Z_{p}$. Если $\varepsilon \neq 0$, то множество $\left\{\varepsilon b^{2}+\varepsilon c^{2}: b, c \in Z_{p}\right\}$ также совпадает с $Z_{p}$, а следовательно, множество чисел вида $a^{2}+\varepsilon b^{2}+\varepsilon c^{2}+d^{2}$ также совпадает с $Z_{p}$. Теперь для доказательства утверждения 2.3 остается лишь заметить, что нормы элементов из $G$ отличны от нуля.

Утверждение 2.4. Центр $\boldsymbol{Z}(G)$ группы $G$ совпадает с множеством элементов $\left\{(a, 0,0,0): a \in \mathbf{Z}_{p} \backslash\{0\}\right\}, u$, следовательно, $|\mathbf{Z}(G)|=p-1$.

Доказательство. Пусть $\alpha=(a, b, c, d) \in Z(G)$. Тогда для любого $\chi=$ $=(x, y, z, w) \in G$ выполняется равенство $\alpha \chi=\chi \alpha$. Вычислив произведения $\alpha \chi, \chi \alpha$ и приравняв компоненты этих векторов, получим систему тождеств относительно $x, y, z, w$ :

$$
d z=c w, d y=b w, \varepsilon c y=\varepsilon b z .
$$

Отметим, что первые компоненты векторов $\alpha \chi, \chi \alpha$ совпадают, и потому значение $a$ может быть произвольным.

Очевидно, что для любых значений $y, z, w$ найдется значение $x$, при котором $\chi \in G$. Значит, равенства (4) должны выполняться при любых $y, z, w$. Очевидно, что при любом фиксированном $\varepsilon$ это возможно лишь при условии $b=c=d=0$. Следовательно, $\alpha=(a, 0,0,0)$, где $a-$ любой элемент из $\mathbf{Z}_{p}$. А так как $\alpha \in G$, то $a \neq 0$, и, значит, $\left\{(a, 0,0,0): a \in \mathbf{Z}_{p} \backslash\{0\} \subset Z(G)\right\}$. Обратное включение очевидно. Утверждение доказано.

Утверждение 2.5. Если $p-1=2^{k} t$, где $t$ нечетно $u k \geq 1$, то множество

$$
N_{s}=\left\{g \in G: N(g)^{2^{s}}=1\right\}
$$

является нормальным делителем группы $G$, и группа $G$ разлагается в произведение $N_{k} \cdot \mathbf{Z}(G)$. 
Доказательство. Из свойства (3) нормы следует, что множество $N_{s}$ образует нормальный делитель группы $G$. Найдем его порядок. Для этого рассмотрим сначала нормальный делитель $N_{0}=\{g \in G: N(g)=1\}$. Легко проверить, что смежными классами группы $G$ по $N_{0}$ являются подмножества элементов с одной и той же нормой. По утверждению 2.3 нормами элементов из $G$ являются все элементы группы $Z_{p}^{*}$. Значит, индекс группы $N_{0}$ в $G$ равен $p-1$ и число элементов с любой фиксированной нормой равно $\frac{|G|}{p-1}$. Так как сравнение $x^{2^{k}} \equiv 1(\bmod p)$ имеет $2^{k}$ различных решений, то из определения множества $N_{k}$ видно, что оно состоит из элементов с $2^{k}$ различными значениями норм. Следовательно,

$$
\left|N_{k}\right|=\frac{2^{k}|G|}{p-1} .
$$

Из утверждения 2.4 следует, что $|Z(G)|=p-1$, причем норма элемента $\alpha=(a, 0,0,0) \in Z(G)$ равна $a^{2}$. Следовательно, $\alpha \in N_{k}$ тогда и только тогда, когда $a^{2^{k+1}}=1$. Так как $\left(2^{k+1}, p-1\right)=2^{k}$, то условию $a^{2^{k+1}}=1$ удовлетворяет ровно $2^{k}$ значений $a$. Следовательно, $\left|N_{k} \cap \mathbf{Z}(G)\right|=2^{k}$ и $\left|N_{k} \cdot \mathbf{Z}(G)\right|=|G|$, поскольку имеет место формула

$$
\left|N_{k} \cdot \mathbf{Z}(G)\right|=\frac{\left|N_{k}\right| \cdot|\mathbf{Z}(G)|}{\left|N_{k} \cap \mathbf{Z}(G)\right|} .
$$

Заметим, что указанное разложение группы $G$ не является ее разложением в прямое произведение.

\section{3. О жордановых формах матриц из группы $\varphi(G)$}

Пусть $\varphi$ - определенное выше матричное представление группы $G$ и $\varphi(\alpha)=A_{\alpha}$.

Известно (см., например, [1]), что жорданова форма $J\left(A_{\alpha}\right)$ матрицы $A_{\alpha}$ для $\alpha=(a, b, c, d) \in H$ определяется по канонической форме $K\left(E x-A_{\alpha}\right)$ матрицы

$$
E x-A_{\alpha}=\left(\begin{array}{cccc}
x-a & \varepsilon b & \varepsilon c & d \\
-b & x-a & d & -c \\
-c & -d & x-a & b \\
-d & \varepsilon c & -\varepsilon b & x-a
\end{array}\right) .
$$


При нахождении матрицы $K\left(E x-A_{\alpha}\right)$ могут возникнуть следующие случаи.

1. $b=c=d=0$. Тогда

$$
E x-A_{\alpha}=\left(\begin{array}{cccc}
x-a & 0 & 0 & 0 \\
0 & x-a & 0 & 0 \\
0 & 0 & x-a & 0 \\
0 & 0 & 0 & x-a
\end{array}\right), J\left(A_{\alpha}\right)=\left(\begin{array}{cccc}
a & 0 & 0 & 0 \\
0 & a & 0 & 0 \\
0 & 0 & a & 0 \\
0 & 0 & 0 & a
\end{array}\right) .
$$

2. $\varepsilon=0, d=0, b c \neq 0, b^{2}+c^{2}=0$. Тогда

$$
K\left(E x-A_{\alpha}\right)=\left(\begin{array}{cccc}
1 & 0 & 0 & 0 \\
0 & x-a & 0 & 0 \\
0 & 0 & x-a & 0 \\
0 & 0 & 0 & (x-a)^{2}
\end{array}\right), J\left(A_{\alpha}\right)=\left(\begin{array}{cccc}
a & 0 & 0 & 0 \\
0 & a & 0 & 0 \\
0 & 0 & a & 1 \\
0 & 0 & 0 & a
\end{array}\right) .
$$

В остальных случаях непосредственными вычислениями находим инвариантные делители $\delta_{i}(x), i=1,2,3,4$, матрицы $E x-A_{\alpha}$ :

$$
\begin{gathered}
\delta_{1}(x)=\delta_{2}(x)=1, \delta_{3}(x)=(x-a)^{2}+\varepsilon b^{2}+\varepsilon c^{2}+d^{2}, \\
\delta_{4}(x)=\left((x-a)^{2}+\varepsilon b^{2}+\varepsilon c^{2}+d^{2}\right)^{2} .
\end{gathered}
$$

Отсюда следует, что

$$
K\left(E x-A_{\alpha}\right)=\left(\begin{array}{cccc}
1 & 0 & 0 & 0 \\
0 & 1 & 0 & 0 \\
0 & 0 & \delta_{3}(x) & 0 \\
0 & 0 & 0 & \delta_{3}(x)
\end{array}\right) .
$$

Заметим, что $\delta_{4}(x)$ есть характеристический многочлен, а $\delta_{3}(x)-$ минимальный многочлен матрицы $A_{\alpha}$.

Теперь в зависимости от свойств многочлена $\delta_{3}(x)$ возможны еще следующие варианты.

3. $\varepsilon b^{2}+\varepsilon c^{2}+d^{2}=0$. В этом случае

$$
E x-A_{\alpha}=\left(\begin{array}{cccc}
1 & 0 & 0 & 0 \\
0 & 1 & 0 & 0 \\
0 & 0 & (x-a)^{2} & 0 \\
0 & 0 & 0 & (x-a)^{2}
\end{array}\right), J\left(A_{\alpha}\right)=\left(\begin{array}{cccc}
a & 1 & 0 & 0 \\
0 & a & 0 & 0 \\
0 & 0 & a & 1 \\
0 & 0 & 0 & a
\end{array}\right) .
$$


4. $r=-\left(\varepsilon b^{2}+\varepsilon c^{2}+d^{2}\right)-$ квадратичный вычет по модулю $p$. Тогда многочлен $\delta_{3}(x)$ разлагается над $Z_{p}$ в произведение двух многочленов первой степени:

$$
\delta_{3}(x)=\left(x-r_{1}\right)\left(x-r_{2}\right), r_{1} \neq r_{2} .
$$

В этом случае

$$
J\left(A_{\alpha}\right)=\left(\begin{array}{cccc}
r_{1} & 0 & 0 & 0 \\
0 & r_{1} & 0 & 0 \\
0 & 0 & r_{2} & 0 \\
0 & 0 & 0 & r_{2}
\end{array}\right), r_{1}, r_{2} \in \mathbf{Z}_{p} .
$$

5. $r=-\left(\varepsilon b^{2}+\varepsilon c^{2}+d^{2}\right)-$ квадратичный невычет по модулю $p$. В этом случае многочлен $\delta_{3}(x)$ неприводим над $\mathbf{Z}_{p}$, а матрица $A_{\alpha}$ не имеет жордановой формы над $\mathbf{Z}_{p}$. Однако матрица $A_{\alpha}$ будет иметь жорданову форму над полем $F$ порядка $p^{2}$, являющимся квадратичным расширением поля $\mathbf{Z}_{p}$, и вид этой формы будет таким же, как и в случае 4 , с той разницей, что $r_{1}, r_{2} \in F$.

Из полученных результатов о нормальных формах матриц $A_{\alpha}$ можно извлечь информацию о порядках элементов группы $\mathrm{G}$ во всех указанных выше случаях 1-5.

Утверждение 3.1.

В случае 1: $\operatorname{ord}(\alpha)=\operatorname{ord}(a)$.

B случае 2: $\operatorname{ord}(\alpha)=p \cdot \operatorname{ord}(a)$.

В случае 3: $\operatorname{ord} \alpha=p \cdot \operatorname{ord}(a)$.

B случае 4: $\operatorname{ord}(\alpha)=\mathrm{HOK}\left(\operatorname{ord}\left(r_{1}\right), \operatorname{ord}\left(r_{2}\right)\right)$.

В случае 5: $\operatorname{ord}(\alpha)$ является делителем числа $p^{2}-1$ и, в частности, совпадает с $p^{2}-1$, если многочлен $\delta_{3}(x)$ примитивен.

Следствие. Порядок любого элемента группы $G$ является делителем числа $p(p-1)$ или числа $p^{2}-1$. В частности, простыми порядками могут быть лишь число $p$ и простые делители чисел $p-1, p+1$.

\section{4. Анализ криптосистемы}

Задача анализа рассмотренной криптосистемы по выработке двумя абонентами общего ключа состоит в следующем.

Исходные известные данные: группа $G$, элементы $g, h \in G$ достаточно больших простых порядков $q_{g}$ и $q_{h}$ соответственно, элементы

$$
u=h^{y_{1}} g^{x_{1}} h^{-y_{1}}, v=h^{y_{2}} g^{x_{2}} h^{-y_{2}} .
$$


Неизвестны числа $x_{1}, x_{2}, y_{1}, y_{2}$.

Требуется найти ключ $K=h^{y_{1}+y_{2}} g^{x_{1} x_{2}} h^{-\left(y_{1}+y_{2}\right)}$. Достаточно найти $x_{1}, x_{2}, y_{1}, y_{2}$.

Ниже будет приведен алгоритм нахождения чисел $x_{1}, x_{2}, y_{1}, y_{2}$.

Искомые числа будем находить из уравнений (5). В связи с этим займемся решением уравнения

$$
h^{y} g^{x} h^{-y}=U
$$

относительно неизвестных $x, y$ при известном $U \in G$.

Сначала найдем неизвестное $x$. Для этого воспользуемся нормой элементов из $G$. Из условия и свойств нормы имеем: $N(U)=$ $=N\left(h^{y} g^{x} h^{-y}\right)=(N(g))^{x}$. Следовательно, $x$ должен быть решением уравнения

$$
(N(g))^{x}=N(U)
$$

в группе $\mathbf{Z}_{p}^{*}$. Однако следует иметь в виду, что в уравнениях (6) и (7) решения находятся лишь с точностью до слагаемых, кратных соответственно порядкам элементов $g$ и $N(g)$. Выясним, как связаны между собой минимальные решения уравнений (6) и (7). Обозначим через $x_{0}, \bar{x}_{0}$ наименьшие неотрицательные решения соответственно уравнений (6), (7). Заметим, что задача нахождения $\bar{x}_{0}$ есть задача дискретного логарифмирования в простом поле $\mathbf{Z}_{p}$. Будем считать, что $\bar{x}_{0}$ уже найден. Очевидно, что $x_{0}<\operatorname{ord}(g)$, $\bar{x}_{0}<\operatorname{ord}_{p}(N(g))$, и множества всех решений этих уравнений можно представить в виде:

$$
M_{0}=\left\{x_{0}+s \cdot \operatorname{ord}(g): s \in \mathbf{Z}\right\}, M_{1}=\left\{\bar{x}_{0}+t \cdot \operatorname{ord}(N(g)): t \in \mathbf{Z}\right\} .
$$

Очевидно также, что $M_{0} \subset M_{1}$, и потому $x_{0}=\bar{x}_{0}+t_{1} \cdot \operatorname{ord}(N(g))$ при некотором $t_{1} \in Z$. Так как отображение $\psi: G \rightarrow \mathbf{Z}_{p}^{*}$ по правилу $\psi(g)=N(g)$ есть эпиморфизм групп, то $\operatorname{ord}(N(g)) \mid \operatorname{ord}(g)$. Таким образом, имеем: $x_{0}=\bar{x}_{0}+t_{1} \cdot \operatorname{ord}(N(g))<\operatorname{ord}(g)$. Отсюда находим: $t_{1}<\frac{\operatorname{ord}(g)-\bar{x}_{0}}{\operatorname{ord}(N(g))}$. А так как $t_{1} \in \mathbf{Z}, \bar{x}_{0}<\operatorname{ord}(N(g))$ и $\operatorname{ord}(N(g)) \mid \operatorname{ord}(g)$, то

$$
t_{1}<\frac{\operatorname{ord}(g)}{\operatorname{ord}(N g))} .
$$


По условию $\operatorname{ord}(g)$ является простым числом, и, следовательно, возможны лишь два варианта: $\operatorname{ord}_{p}(N(g))=\operatorname{ord}(g)$ или $\operatorname{ord}(N(g))=1$. В первом варианте $t_{1}=0$ и $x_{0}=\bar{x}_{0}$. Поэтому остается рассмотреть лишь второй вариант, в котором $\operatorname{ord}(N(g))=1$, а потому и

$$
N(g)=1 .
$$

При рассмотрении этого варианта мы воспользуемся найденными выше жордановыми формами элементов $g, U \in G$ из уравнения (6).

Пусть $g=(a, b, c, d)$. Рассмотрим указанные в предыдущем пункте 5 случаев для элемента $\alpha=g$ и соответствующей ему матрицы $A_{g}$.

1. Здесь $\operatorname{ord}(g)=\operatorname{ord}(a)=q_{g}, N(g)=a^{2}, \operatorname{ord}(N(g))=\operatorname{ord}\left(a^{2}\right)$. Так как число $q_{g}$ нечетно, то $\operatorname{ord}\left(a^{2}\right)=\operatorname{ord}(a)$, и, следовательно, $\operatorname{ord}(g)=\operatorname{ord}(N(g))$.

2. Здесь $\quad \operatorname{ord}(g)=p \cdot \operatorname{ord}(a), \quad N(g)=a^{2}, \quad \operatorname{ord}(N(g))=\operatorname{ord}\left(a^{2}\right)=\operatorname{ord}(a)$, и в силу условия (8) возможен лишь один вариант: ord $g=p, a=1$. В этом случае

$$
J\left(A_{g}\right)=\left(\begin{array}{cccc}
1 & 0 & 0 & 0 \\
0 & 1 & 0 & 0 \\
0 & 0 & 1 & 1 \\
0 & 0 & 0 & 1
\end{array}\right) \text { и } J\left(A_{g}^{x}\right)=\left(\begin{array}{cccc}
1 & 0 & 0 & 0 \\
0 & 1 & 0 & 0 \\
0 & 0 & 1 & x \\
0 & 0 & 0 & 1
\end{array}\right) .
$$

Из (6) видно, что матрицы $A_{g}^{x}$ и $A_{U}$ подобны, и потому их жордановы формы совпадают. Следовательно, жорданова форма матрицы $A_{U}$ имеет вид

$$
J\left(A_{U}\right)=\left(\begin{array}{llll}
1 & 0 & 0 & 0 \\
0 & 1 & 0 & 0 \\
0 & 0 & 1 & r \\
0 & 0 & 0 & 1
\end{array}\right)
$$

при некотором $r \in \mathbf{Z}_{p}$. Отсюда получаем $x=r$.

3. Здесь аналогичными рассуждениями приходим к единственно возможному случаю $\operatorname{ord} g=p, a=1$, и тогда

$$
J\left(A_{g}^{x}\right)=\left(\begin{array}{llll}
1 & x & 0 & 0 \\
0 & 1 & 0 & 0 \\
0 & 0 & 1 & x \\
0 & 0 & 0 & 1
\end{array}\right), J\left(A_{U}\right)=\left(\begin{array}{llll}
1 & r & 0 & 0 \\
0 & 1 & 0 & 0 \\
0 & 0 & 1 & r \\
0 & 0 & 0 & 1
\end{array}\right), x=r .
$$


4. Здесь $\operatorname{ord}(g)=$ НОК $\left(\operatorname{ord}\left(r_{1}\right), \operatorname{ord}\left(r_{2}\right)\right)$. Найдем $N(g)$. С одной стороны, $\delta_{3}(x)=(x-a)^{2}+\varepsilon b^{2}+\varepsilon c^{2}+d^{2}=(x-a)^{2}+N(g)-a^{2}=x^{2}-2 a x+N(g)$, a с другой стороны, $\delta_{3}(x)=\left(x-r_{1}\right)\left(x-r_{2}\right)=x^{2}-\left(r_{1}+r_{2}\right)+r_{1} r_{2}$. Отсюда следует, что $N(g)=r_{1} r_{2}$, и по условию (7) $r_{2}=r_{1}^{-1}$. Поэтому $\operatorname{ord}(g)=\operatorname{ord}\left(r_{1}\right)=$ $=\operatorname{ord}\left(r_{2}\right)=q_{g}$, и

$$
J\left(A_{g}^{x}\right)=\left(\begin{array}{cccc}
r_{1}^{x} & 0 & 0 & 0 \\
0 & r_{1}^{x} & 0 & 0 \\
0 & 0 & r_{1}^{-x} & 0 \\
0 & 0 & 0 & r_{1}^{-x}
\end{array}\right), J\left(A_{U}\right)=\left(\begin{array}{cccc}
w & 0 & 0 & 0 \\
0 & w & 0 & 0 \\
0 & 0 & w^{-1} & 0 \\
0 & 0 & 0 & w^{-1}
\end{array}\right) .
$$

Следовательно, $x$ находится из уравнения $r_{1}^{x}=w$ в группе $\mathbf{Z}_{p}^{*}$ при дополнительной информации $\operatorname{ord}\left(r_{1}\right)=q_{g}$ и $q_{g} \mid p-1$.

5. В этом случае матрица $A_{g}$ не имеет жордановой формы над $\mathbf{Z}_{p}$. Однако она будет иметь жорданову форму над расширением $F$ второй степени поля $\mathbf{Z}_{p}$. Причем она будет иметь такой же вид, как и в случае 4 , с той лишь разницей, что $r_{1}, r_{2}$ будут элементами из $F \backslash \mathbf{Z}_{p}$. При этом $\operatorname{ord}(g) \mid p^{2}-1$, и, значит, $q_{g} \mid p-1$ или $q_{g} \mid p+1$. Из вида жордановой формы матрицы $A_{g}$ следует, что $\operatorname{ord}(g)=\operatorname{ord}\left(r_{1}\right)$. Следовательно, при условии $q_{g} \mid p-1$ элементы $r_{1}, r_{2}$ будут содержаться в $\mathbf{Z}_{p}$, что противоречит условию. Значит, $q_{g} \mid p+1$ и $x$ будет находиться из уравнения $r_{1}^{x}=w$ в группе $F^{*}$ при дополнительном условии $\operatorname{ord}\left(r_{1}\right)=q_{g}$ и $q_{g} \mid p+1$.

Выясним, для каких элементов $g=(a, b, c, d)$ имеет место случай 5 . Из приведенных рассуждений следует, что случай 5 возможен тогда и только тогда, когда выполняется условие (8) и многочлен $\delta_{3}(x)=$ $=(x-a)^{2}+\varepsilon b^{2}+\varepsilon c^{2}+d^{2}$ неприводим над $Z_{p}$. Из условия (8) следует, что

$$
\delta_{3}(x)=x^{2}-2 a x+1=(x-a)^{2}-\left(a^{2}-1\right) .
$$

Отсюда видно, что $\delta_{3}(x)$ неприводим в том и только том случае, когда $a^{2}-1$ является квадратичным невычетом по модулю $p$.

Таким образом, сложность проблемы нахождения показателя $x$ из уравнения (6) не превосходит по порядку сложность проблемы логарифмирования в простом поле $\mathbf{Z}_{p}$ либо в его квадратичном расширении.

Пусть, решая уравнения (5), мы нашли $x_{1}, x_{2}$ и затем вычислили $g^{x_{1}}, g^{x_{2}}$. 
Рассмотрим теперь задачу нахождения неизвестных $y_{1}, y_{2}$. Для этого вернемся к решению уравнения (6), предполагая, что уже найдены значение неизвестного $x$ и элемент $\beta=g^{x}$.

По утверждению 2.5 элемент $h$ можно представить в виде произведения элементов $u, v$ из подгрупп $N_{k}$ и $\mathbf{Z}(G)$ соответственно. Тогда уравнение (6) равносильно уравнению, в котором вместо $h$ выступает $u$. Следовательно, не теряя общности, будем считать, что в уравнении (6) $h \in N_{k}$, где $k$ - максимальное натуральное число, при котором $2^{k} \mid p-1$.

Пусть $h=(a, b . c . d)$ и $A_{h}=T J\left(A_{h}\right) T^{-1}$. Тогда уравнение (6) эквивалентно уравнению

$$
J\left(A_{h}\right)^{y} C=D J\left(A_{h}\right)^{y},
$$

где $C=T^{-1} A_{\beta} T, D=T^{-1} A_{U} T$.

При нахождении неизвестного $y$ мы будем пользоваться жордановой формой матрицы $A_{h}$, рассматривая указанные выше 5 случаев.

1. Этот случай не представляет интереса, поскольку в нем $g$ принадлежит центру группы $G$, тогда как по условию $h$ и $g$ не содержатся в $Z(G)$.

2. Здесь

$$
J\left(A_{h}\right)=\left(\begin{array}{llll}
a & 0 & 0 & 0 \\
0 & a & 0 & 0 \\
0 & 0 & a & 1 \\
0 & 0 & 0 & a
\end{array}\right) \text { и } N(h)=a^{2} .
$$

Так как $h \in N_{k}$, то $N(h)^{2^{k}}=\left(a^{2}\right)^{2^{k}}=a^{2^{k+1}}=1, \quad$ и, следовательно, $\operatorname{ord}(N(h))=2^{t}, \operatorname{ord}(a)=2^{t+1}, 0 \leq t \leq k$. А так как

$$
J\left(A_{h}\right)^{y}=\left(\begin{array}{cccc}
a^{y} & 0 & 0 & 0 \\
0 & a^{y} & 0 & 0 \\
0 & 0 & a^{y} & y a^{y-1} \\
0 & 0 & 0 & a^{y}
\end{array}\right),
$$

то $\operatorname{ord}(h)=p \cdot \operatorname{ord}(a)=p \cdot 2^{t+1}$. По условию $\operatorname{ord}(h)=q_{h}-$ простое число, что возможно лишь при условии $\operatorname{ord}(h)=p, \operatorname{ord}(a)=1$. Поэтому $a=1$ и

$$
J\left(A_{h}\right)^{y}=\left(\begin{array}{cccc}
1 & 0 & 0 & 0 \\
0 & 1 & 0 & 0 \\
0 & 0 & 1 & y \\
0 & 0 & 0 & 1
\end{array}\right)
$$


Подставив матрицу $J\left(A_{h}\right)^{y}$ в уравнение (9), получим систему линейных уравнений с одним неизвестным, которая решается тривиальным образом.

3. В этом случае теми же рассуждениями, что и в случае 2 , приходим к матрице

$$
J\left(A_{h}\right)^{y}=\left(\begin{array}{llll}
1 & y & 0 & 0 \\
0 & 1 & 0 & 0 \\
0 & 0 & 1 & y \\
0 & 0 & 0 & 1
\end{array}\right)
$$

а затем к системе линейных уравнений с одним неизвестным $y$.

4. В этом случае

$$
J\left(A_{h}\right)=\left(\begin{array}{cccc}
r_{1} & 0 & 0 & 0 \\
0 & r_{1} & 0 & 0 \\
0 & 0 & r_{2} & 0 \\
0 & 0 & 0 & r_{2}
\end{array}\right) \text { и } J(h)^{y}=\left(\begin{array}{cccc}
r_{1}^{y} & 0 & 0 & 0 \\
0 & r_{1}^{y} & 0 & 0 \\
0 & 0 & r_{2}^{y} & 0 \\
0 & 0 & 0 & r_{2}^{y}
\end{array}\right) .
$$

Здесь $\operatorname{ord}(h)=\mathrm{HOK}\left(\operatorname{ord}\left(r_{1}\right), \operatorname{ord}\left(r_{2}\right)\right), N(h)=r_{1} r_{2}, \operatorname{ord}(N(h))=\operatorname{ord}\left(r_{1} r_{2}\right)$. Отсюда, учитывая, что $\operatorname{ord}(h)-$ простое число, $h \in N_{k}$ и $\operatorname{ord}(N(h)) \mid \operatorname{ord}(h)$, получим $\operatorname{ord}(h)=\operatorname{ord}\left(r_{1}\right)=\operatorname{ord}\left(r_{2}\right)=q_{h}, \operatorname{ord}\left(r_{1} r_{2}\right)=2^{t}, 0 \leq t \leq k \quad$ и $2^{t} \mid q_{h}$. Следовательно, $t=0, r_{1} r_{2}=1$, и

$$
J\left(A_{h}\right)^{y}=\left(\begin{array}{cccc}
u & 0 & 0 & 0 \\
0 & u & 0 & 0 \\
0 & 0 & v & 0 \\
0 & 0 & 0 & v
\end{array}\right) \text {, где } u=r_{1}^{y}, v=r_{1}^{-y}
$$

Подставив матрицу $J\left(A_{h}\right)^{y}$ в уравнение (9), получим систему линейных уравнений с двумя неизвестными $u, v$. При ее решении следует учесть также, что $u \cdot v=1$. Далее, имея $u$, искомое неизвестное $y$ найдем из уравнения $u=r_{1}^{y}$ при дополнительном условии $y<q_{h}, q_{h} \mid p-1$.

5. В этом случае $y$ находится точно так же, как и в случае 4 , разница лишь в том, что $r_{1}$ будет принадлежать расширению $F$ поля $\mathbf{Z}_{p}$ и уравнение $r_{1}^{y}=u$ придется решать над полем $F$ при условии $y<q_{h}, \operatorname{ord}\left(r_{1}\right)=q_{h}$, $q_{h} \mid p+1$.

В итоге доказано следующее утверждение. 
Теорема. Верхняя оченка криптографической стойкости рассмотренной криптосистемы открытого распределения ключей по порядку не превосходит сложности проблемы дискретного логарифмирования в ичклической подгруппе порядка q мультипликативной группы поля $\mathbf{Z}_{p}$ или его квадратичном расширении, где q- простой делитель соответственно чисел $p-1$ или $p+1$.

ЗАМЕЧАНИЕ. Проведенный здесь анализ криптосистемы применим ко всем системам с некоммутативными группами, определенными в работах $[4,5,8,9]$ на 4-мерных векторах над полем $\mathbf{Z}_{p}$. Итоговый результат будет таким же. Суть в том, что во всех этих случаях построенные группы изоморфны подходящим группам матриц размеров $4 \times 4$ над полем $\mathbf{Z}_{p}$, причем характеристические многочлены используемых матриц разлагаются над полем $\mathbf{Z}_{p}$ в произведение многочленов не выше второй степени.

\section{Список литературы}

1. Глухов М. М., Елизаров В. П., Нечаев А. А. Алгебра. Т. ІІ. - М., Гелиос APB, 2003.

2. Сидельников В. М., Черепнев М. А., Ященко В. В. Системы открытого распределения ключей на основе некоммутативных полугрупп. - Докл. PAH, 1993, т. 332, № 5, с. 566-567.

3. Сиделъников В. М. Системы распределения ключей на основе «экспоненциального представления» линейной группы $G L_{n}\left(F_{p}\right) .-$ Проблемы передачи информации, 1994, т. 30, в. 4, с. 25-32.

4. Молдовян Н. А. Теоретический минимум и алгоритмы цифровой подписи. - БХВ-Петербург, 2010.

5. Молдовян Д. Н., Куприянов А. И., Костина А. А., Захаров Д. В. Задание некоммутативных конечных групп векторов для синтеза алгоритмов цифровой подписи. - Вопросы защиты информации, 2009, № 4, с. 12-18.

6. Anshel I., Anshel M., Goldfeld D. An algebraic method for public key cryptography. - Math. Res. Lett., 1999, v. 6, p. 287-291.

7. Koo K. H., Lee S. J., Cheon J. H., Han J. W., Kang J., Park C. New publickey cryptosystem using braid groups. - CRYPTO'2000, Lect. Notes Comput. Sci., 2000, v. 1880, p. 166-183.

8. Moldovyan N. A., Moldovyanu P. A. New primitives for digital signature algorithms: vector finite fields. - Quasigroups and related systems, 2009, v. 17, p. 271-282.

9. Moldovyan D. N., Moldovyan N.A. A new hard problem over noncommutative finite groups for cryptographic protocols. - MMM-ACNS 2010, p. 183-194. 
10. Paeng S. H., Ha K. C., Kim J. H., Chee S., Park C. New public key cryptosystem using finite non abelian groups. - CRYPTO'2001, Lect. Notes Comput. Sci., 2001, v. 2139, p. 470-485.

11. Paeng S. H., Kwon D., Ha K. C., Kim J. H. Improved public key cryptosystem using finite non abelian groups. - http://eprint.iacr.org/2001/066.

12. Sakalauskas E., Tvarijonas P., Raulynaitis A. Key agreement protocol (KAP) using conjugacy and discrete logarithms problems in group representation level. - Informatica, 2007, v. 18, № 1, p. 115-124.

13. Shor $P$. $W$. Polynomial-time algorithm for prime factorization and discrete logarithms on quantum computer. - SIAM J. Comput., 1997, v. 26, p. 14841509.

14. Tobias $C$. Security analysis of MOR using GL $(2, \mathrm{R}) \times_{\text {Theta }} Z_{\mathrm{n}} .-2^{\text {nd }}$ Int. Workshop Secur. Inform. Syst., WOSIS 2004, p. 170-179.

15. Tobias $C$. Design und Analyse kryptografischer Bausteine auf nicht-abelschen Gruppen. - Diss. Dokt. Naturwiss., Giessen, 2004, 128 pp.

16. Yamamura A. Public key cryptosystems using the modular group. - PKC, Lect. Notes Comput. Sci., 1998, v. 1431, p. 203-216.

17. Yamamura A. A functional cryptosystems using a group action. - ACISP, Lect. Notes Comput. Sci., 1999, v. 1587, p. 314-325. 

